\title{
EL ASALTO DE TRUMP AL DERECHO INTERNACIONAL: SU IMPACTO EN LA OMC
}

\author{
TRUMP'S ASSAULT ON INTERNATIONAL LAW: ITS IMPACT ON THE WTO*
}

\section{Ricardo Arredondo*}

\section{RESUMEN}

La asunción al poder del presidente de los Estados Unidos Donald J. Trump significó un giro radical tanto en el plano interno como en el internacional, que contribuyó a exacerbar las divisiones en la política doméstica estadounidense, a la vez que trajo aparejadas importantes consecuencias negativas para el multilateralismo y los regímenes jurídicos internacionales vigentes. Este ataque, que se manifiesta en diferentes áreas del derecho internacional, tiene entre sus objetivos a diversas organizaciones internacionales, cuya muestra más reciente ha sido la suspensión de fondos a la Organización Mundial de la Salud (OMS) en medio de la pandemia del coronavirus. La Organización Mundial de Comercio no solo no ha escapado a esta embestida, sino que ha sido uno de los blancos particularmente elegidos por la administración estadounidense. Este trabajo analiza el ataque de Trump a la OMC, sus consecuencias y las posibles salidas a este impasse.

Palabras clave: derecho internacional, organizaciones internacionales, OMC, solución de diferencias, Estados Unidos, multilateralismo.

\section{ABSTRACT \\ Since Donald Trump came to power, the United States has been going through one of the most turbulent periods in its history, which has been manifested in a deep internal political rift and, externally, in an assault on international law. This attack, which manifests itself in different areas of international law, has among its objectives various international organizations, the most recent example of which has been the suspension of funds to the World Health Organization (WHO) in the midst of the coronavirus pandemic. The World Trade Organization has not only not escaped}

\footnotetext{
* Abogado (Universidad Nacional de Tucumán), Master of Laws (LL.M) (LSE) y Doctor en Derecho (UBA). Profesor de Derecho Internacional Público (UBA y Univ. de Palermo). Profesor de Derecho y Práctica Diplomática (ISEN). Profesor de Teoría y Práctica Diplomática y Consular (Universidad de Belgrano), Profesor de International Human Rights Law (Universidad Austral). Miembro del Instituto de Derecho Internacional de la Academia Nacional de Derecho y Ciencias Sociales de Buenos Aires, de la Asociación Argentina de Derecho Internacional y del Instituto de Derecho Internacional del CARI, entre otras instituciones.

Este trabajo es una versión actualizada de la ponencia presentada por el autor en el XXXI Congreso de la Asociación Argentina de Derecho Internacional, llevado a cabo en Córdoba, Argentina, del 4 al 7 de septiembre de 2019. A su vez, forma parte de un proyecto más amplio titulado "El asalto de Trump al multilateralismo y al Derecho internacional. Posibles cursos de acción para una política exterior argentina", con el auspicio de la Universidad de Palermo. El autor desea agradecer especialmente los valiosos comentarios de Pedro F. Negueloaetcheverry, que contribuyeron a mejorar la versión inicial de este trabajo.
} 
this onslaught, but has been one of the targets particularly chosen by the US administration. This paper analyzes Trump's attack on the WTO, its consequences and possible solutions to this impasse.

Keywords: international law, international organizations, WTO, dispute settlement, United States, multilateralism.

\section{Introducción}

La asunción al poder del presidente de los Estados Unidos Donald J. Trump significó un giro radical tanto en el plano interno como en el internacional, que contribuyó a exacerbar las divisiones en la política doméstica estadounidense, a la vez que trajo aparejadas importantes consecuencias negativas para el multilateralismo y los regímenes jurídicos internacionales vigentes.

La pandemia del coronavirus ha venido a acelerar el proceso de fragmentación del orden internacional creado con posterioridad a la segunda guerra mundial, que venía sufriendo ataques derivados tanto de factores externos (el reposicionamiento de China y su lucha por la primacía del poder mundial, el resurgimiento de Rusia luego de los años de ostracismo posteriores a finalización de la Guerra Fría y el fallido intento democratizador conocido como "Primavera Árabe", entre otros), como internos o propios de ese orden, llamado liberal por algunos, que se evidenciaban en un escepticismo respecto de sus valores y principios fundadores -incluida la pérdida de fe en la democracia (Connaughton et al., 2020)-, la aparición de movimientos antidemocráticos inclusive al interior de la Unión Europea -v.g. Polonia, Hungría-, el ascenso de corrientes populistas y tendencias nacionalistas como el Brexit ${ }^{1}$. Un elemento sustantivo entre ese conjunto de negatividades es el asalto de Trump al derecho internacional. No es un secreto que el orden internacional liberal viene siendo desafiado por una serie de factores tanto exógenos (el ascenso de China en el orden global, la política exterior rusa y el fracaso de la "Primavera Árabe", por mencionar algunos) como endógenos, entendidos éstos como una afrenta a los valores liberales en el seno de Occidente, que se manifiesta en un proceso de auténtica implosión de este orden (pérdida de fe en los valores centrales del liberalismo, ascenso del populismo tanto de derecha como de izquierda, surgimiento de movimientos fuertemente nacionalistas como Brexit). Entre esas fuerzas internas que están afectando seriamente el orden mundial basado en normas sobresale claramente el ataque de Trump al derecho internacional.

Trump no es la causa única ni principal de los cambios en el escenario internacional, sino que ellos responden a fuerzas económicas y sociales más profundas, "de las que el propio Trump es una consecuencia" (Sanahuja, 2020, 81). La embestida parece ser impulsada por una combinación de factores como nacionalismo económico, antiglobalismo ${ }^{2}$, anti-elitismo, la creencia de que el derecho internacional no refleja los valores estadounidenses, sino que amenaza a sus instituciones,

\footnotetext{
${ }^{1}$ Para un análisis más detallado del impacto de los movimientos nacionalistas véase Tamir, 2020.

2 Debe hacerse una distinción entre los conceptos "antiglobalismo" y "antiglobalización", en la medida en que el primero representa un enfrentamiento al sistema multilateral basado en normas, como en el caso de los Estados Unidos (véase Slobodian, 2018); mientras que el segundo expresa una crítica social al proceso de globalización, como en el caso de la India (véase Bhagwati, 2002).
} 
y una afirmación continua y constante de que la paz, el prestigio y la prosperidad estadounidenses están siendo desatendidos por su política exterior.

Koh describe a la administración Trump como un período tumultuoso que ha afectado de manera negativa muchos factores del orden mundial global, incluido el derecho internacional, y se pregunta si estas presiones se traducirán en un cambio permanente en la relación de los Estados Unidos con el derecho y las instituciones internacionales (Koh, 2019). El asalto de Trump al derecho internacional implica abandonar la visión kantiana de un orden internacional basado en normas, que, aunque imperfecto, ha funcionado razonablemente bien. De triunfar esta cosmovisión trumpiana, el mundo se enfrentaría a un sistema más cínico, orwelliano, de gobierno global, dominado por grandes potencias realistas que ejercerían su poder de sus zonas de influencia. Así, la filosofía del "América primero" de Trump actúa en función de un auto-percibido interés nacional, donde las normas internacionales brillan por su ausencia.

\section{La Administración Trump y las instituciones multilaterales}

La desconfianza, cuando no el abierto ataque, a las organizaciones internacionales ha sido una marca registrada de los Estados y, en particular del Partido Republicano, al menos desde fines de la década de 1950, cuando el proceso de descolonización transformó a la Asamblea General de las Naciones Unidas de ser un órgano pro-occidental en su mayoría, que votaría a favor de los Estados Unidos, en uno heterogéneo y diverso que en diversas ocasiones pondría objeciones y criticaría abiertamente su accionar (Pearson y Rochester, 2000, 148). Esa susceptibilidad se tradujo en una presión de los Estados Unidos sobre los órganos de las instituciones multilaterales para que adoptaran decisiones o resoluciones que favorecieran sus intereses, bajo la amenaza de recortar sus fondos y, eventualmente, retirarse de las organizaciones por completo (Foot et al., 2003).

La noción de que las organizaciones internacionales atentan contra la soberanía estadounidense ha sido durante décadas un pilar del conservadurismo estadounidense. La plataforma del Partido Republicano de 1984 lo expresaba cuando afirmaba que los estadounidenses no pueden contar con las organizaciones internacionales para garantizar su seguridad o proteger adecuadamente sus intereses y, por lo tanto, no apoyarían organizaciones internacionales incompatibles con esos intereses (The American Presidency Project, 1984). En la década de los 80s, el gobierno de los Estados Unidos se negó a ratificar la Convención de Naciones Unidas sobre Derecho del Mar, retiró a los Estados Unidos de la Organización de las Naciones Unidas para la Educación, la Ciencia y la Cultura (UNESCO), a la que luego regresó para posteriormente volver a retirarse durante la administración Trump, y se mantuvo sin pagar su cuota a las Naciones Unidas hasta que en 1997 regularizó su situación. En 1995, el legislador de Florida, Joe Scarborough, presentó un proyecto de ley denominado "Ley de Retiro de las Naciones Unidas" (Scarborough, 1995), cuyo objetivo era que Estados Unidos se retirara por completo de la ONU y se trasladara la sede de organización fuera de los Estados Unidos. Ese proyecto no pasó de la fase de comité (Baron, 2020).

Esa animadversión hacia las Naciones Unidas y otras instituciones multilaterales se ha profundizado y ha tomado un mayor alcance y significado desde entonces. La extrema derecha, aislacionista-nacionalista, al estilo de John Bolton y conservadores como Trump, han criticado abiertamente al multilateralismo y la globalización, incluyendo obviamente a las instituciones 


\section{Revista Peruana de Derecho Internacional \\ Tomo LXX Mayo-Agosto $2020 \mathcal{N}^{\circ} 165$, pp. 197-225. ISSN: 2663-0222 \\ Recepción: 30/05/2020 Aceptación: 07/07/2020}

internacionales, en la medida que los consideran "obstáculos" para que los Estados Unidos puedan imponer sus puntos de vista en el escenario internacional (Khaler, 2017).

La relación de desdén del presidente Trump con el derecho y las instituciones internacionales no comenzó cuando asumió la presidencia, sino que viene desde antes y se reveló ya con claridad durante su campaña presidencial, en su discurso inaugural en enero de 2017 (White House, 2017), y persistentemente desde entonces. A título de ejemplo puede señalarse que, en su discurso ante la Asamblea General de las Naciones Unidas, en septiembre de 2018, expresó que los Estados Unidos siempre elegirá la independencia y la cooperación sobre la gobernanza global, el control y la dominación (White House, 2018), en lo que Goldschmidt califica como una formulación "típica" del presidente estadounidense (Goldschmidt y Mercer, 2018). Asimismo, disconforme con la membresía y las acciones adoptadas por el Consejo de Derechos Humanos de las Naciones Unidas, retiró a los Estados Unidos de este órgano sustancial del sistema multilateral (US Department of State, 2018 y Galbraith, 2018, 745-751), dejó de aportar fondos para el Organismo de Obras Públicas y Socorro de las Naciones Unidas para los Refugiados de Palestina en el Cercano Oriente (UNWRA), redujo su contribución a la Organización de Estados Americanos (OEA) debido a la posición de la Comisión Interamericana de Derechos Humanos en materia de salud reproductiva (US Department of State, 2019), se retiró del Acuerdo de París sobre Cambio Climático (Arredondo, 2018, 61), entre muchas otras acciones tendientes a minar las instituciones multilaterales y la gobernanza global. El más reciente blanco de esos ataques ha sido la Organización Mundial de la Salud (OMS) (Noack, 2020). Descontento con la acción de la OMS, a la que acusa de corrupta y de haber obrado de manera negligente frente a la aparición de la enfermedad del COVID-19, primero anunció la suspensión de fondos y ahora directamente ha expresado su decisión de retirar a los Estados Unidos de la OMS en medio de una de las más graves pandemias que ha asolado el planeta. Un movimiento que adversarios como Putin $\mathrm{y}$, particularmente, Xi Jinping deben haber recibido con una sonrisa en los labios.

\section{La diplomacia comercial de la Administración Trump y el sistema multilateral de comercio}

En particular, la "diplomacia comercial" de los Estados Unidos, es un claro ejemplo donde la administración ejerció presión, agrietó alianzas y generó "guerras comerciales". Apenas asumido su mandato, Trump anunció que los Estados Unidos no serían parte del Acuerdo Comercial Transpacífico (White House, 2017). Ello le fue facilitado por el hecho de que el Congreso no había manifestado su consentimiento para obligarse por el TTP y, por lo tanto, los Estados Unidos no habían asumido obligaciones en virtud del acuerdo. Trump presumía que, sin su presencia, el acuerdo no seguiría adelante (Koh, 2019, 54). La realidad lo mostró equivocado una vez más. Las restantes partes del TPP luego negociaron y, en marzo de 2018, firmaron un acuerdo similar conocido como el Tratado Integral y Progresista de Asociación Transpacífico (CPTPP, por sus siglas en inglés) (OEA-SICE, 2019). En abril de 2018, Trump indicó que consideraría unirse al TPP, pero luego criticó rápidamente el acuerdo (Trump, 2018). Hasta el momento, Estados Unidos 
no ha tomado medidas oficiales para unirse al CPTPP, aunque ha celebrado nuevos acuerdos comerciales bilaterales con Corea del $\operatorname{Sur}^{3}$ y con Japón. ${ }^{4}$

También procuró retirarse del Tratado de Libre Comercio de las Américas (NAFTA, por sus siglas en inglés), con el alegado objetivo de alcanzar mejores condiciones para los trabajadores estadounidenses y que, si no podía conseguir un mejor trato, se retiraría del acuerdo (Trump, 2016). Sin embargo, ante la presión de Departamento de Agricultura, se vio obligado a dar marcha atrás y renegociar el tratado con sus vecinos y socios comerciales. Las renegociaciones comenzaron en enero de 2018 y, el 30 de noviembre de 2018, las partes firmaron un nuevo tratado llamado Acuerdo Estados Unidos, Canadá y México (USMCA, por sus siglas en inglés) (Landler y Rappeport, 2018). ${ }^{5}$

Asimismo, inició una serie de conflictos comerciales con muchos de sus socios, incluida China, India y otros, para corregir prácticas comerciales supuestamente injustas, invocando cuestiones de "seguridad nacional". Cabe recordar que el Art. XXI del Acuerdo General sobre Aranceles Aduaneros y Comercio (GATT, 1947) contiene excepciones a la aplicación del acuerdo basadas en "cuestiones de seguridad", que, entre cosas, permiten "a una parte contratante la adopción de todas las medidas que estime necesarias para la protección de los intereses esenciales de su seguridad". Existe consenso en señalar que esta disposición es auto evaluadora o juzgadora, lo que significa que los Estados pueden decidir cuándo la invocarán (Alford, 2011, 698). El Artículo XXI del GATT rara vez ha sido invocado y "cada vez que se mencionó como justificante de las medidas adoptadas por las Partes Contratantes del GATT, o por los Miembros de la OMC, suscitó fuertes reacciones, tanto a favor como en contra" (Vásquez Arango, 2011). El asunto DS512: RusiaMedidas que afectan al tráfico en tránsito fue la primera ocasión, desde la creación de la OMC, en que un Miembro de la organización invocó esta disposición en una disputa. Por lo tanto, esta fue también la primera vez que se le pidió a un Grupo Especial (panel) de la OMC que interpretara el significado del artículo XXI, incluidas las preguntas clave sobre si la frase "que estime necesarias" significa que la disposición es "autoevaluada" por el Miembro de la OMC invocando la excepción, o si otros elementos del tex to del Artículo XXI califican (y, por lo tanto, limitan) la naturaleza de juicio de la disposición (Barshefsky et al., 2019). El Grupo Especial rechazó el argumento ruso (y estadounidense) de que el artículo no puede ser objeto de revisión por un panel, aunque finalmente consideró que las acciones rusas eran consistentes con la excepción de seguridad nacional: "Rusia ha satisfecho las condiciones de la parte introductoria del apartado b) del artículo XXI del GATT de 1994; y por consiguiente, Rusia ha cumplido los requisitos necesarios para invocar el inciso iii) del apartado b) del artículo XXI en relación con las medidas en litigio y, en consecuencia, las medidas en litigio están abarcadas por el inciso iii) del apartado b) del artículo XXI del GATT de 1994" (OMC Doc. WT/DS512/R, para. 8.2.d), p. 116). El informe del panel también encontró que los tres subpárrafos de la sección b) que establecen las

\footnotetext{
${ }^{3}$ En el caso de Corea se trata de la renegociación de un acuerdo de libre comercio existente entre ambos países, conocido comúnmente como KORUS, que entró en vigor el 1 de enero de 2019 (Cfr. Lester, Manak y Kim, 2019).

${ }^{4}$ El presidente de los Estados Unidos, Donald J. Trump, y el primer ministro japonés, Shinzo Abe, anunciaron en septiembre de 2019 un acuerdo limitado para reducir los aranceles y otras barreras comerciales entre sus países, que podría entrar en vigor en enero de 2020. Trump calificó el acuerdo como "fenomenal", pero los expertos en comercio y algunos miembros del Congreso estadounidense siguen siendo escépticos al respecto (Cfr. Chatzky, 2019).

${ }^{5}$ El texto completo del tratado puede verse en https://ustr.gov/trade-agreements/free-trade-agreements/united-statesmexico-canada-agreement/agreement-between.
} 


\section{Revista Peruana de Derecho Internacional \\ Tomo LXX Mayo-Agosto $2020 \mathcal{N}^{\circ}$ 165, pp. 197-225. ISSN: 2663-0222 \\ Recepción: 30/05/2020 Aceptación: 07/07/2020}

circunstancias en las que un Miembro puede invocar una excepción de seguridad nacional pueden ser consideradas objetivamente. Asimismo, el panel afirmó que las medidas justificadas por la excepción de seguridad nacional pueden revisarse para determinar si son necesarias para proteger la seguridad nacional y si tienen una conexión plausible con el interés de seguridad nacional mencionado por el demandado en una disputa. En síntesis, el panel encontró que puede revisar una medida que un Miembro de la OMC alega que "estime necesarias" para proteger su propio interés de seguridad porque puede determinar objetivamente si una de las tres circunstancias establecidas en los subpárrafos de la sección b) del artículo XXI del GATT de 1994 ocurrió en el momento de la imposición de la medida y porque puede determinar objetivamente si la medida tiene una conexión plausible con la circunstancia identificada (Reinsch y Caporal, 2019).

Sin embargo, en muchos supuestos, la propia imbricación de la política comercial externa de los Estados Unidos con sistema multilateral de comercio ha obstaculizado la salida de estos acuerdos comerciales, con lo cual la política de Trump solo ha conseguido alienar a los socios comerciales sin crear otros nuevos, contribuyendo de esta manera a perjudicar la propia posición de los Estados Unidos (Koh, 2019, 58). En particular, Koh señala que en América Latina la postura de Trump "irónicamente" ha hecho más que ningún otro factor económico para favorecer la integración comercial en oposición a sus iniciativas (Koh, 2019, 60).

El sistema multilateral de comercio se enfrenta a uno de los escenarios más adversos de sus 25 años de existencia, con una profunda crisis interna marcada por el proteccionismo. Aunque abundan los titulares de aumentos de los aranceles multimillonarios a las exportaciones chinas con su consiguiente retaliación a las exportaciones estadounidenses, de hecho, desde noviembre de 2008, 36 gobiernos llevaron a cabo un total de 348 distorsiones en el comercio, cada una de las cuales afectó a más de $\$ 10$ mil millones de dólares. Catorce gobiernos son responsables de imponer cinco o más medidas proteccionistas de este tipo. Solo 6 de estas 348 medidas proteccionistas gigantes han sido implementadas por la Administración de Trump. "La podredumbre comenzó mucho antes de la guerra comercial chino-estadounidense" (Evenett y Fritz, 2019, 6).

Es más, el 1 de agosto de 2019, Trump aumentó la tensión con China al anunciar que, a partir del 1 de septiembre de 2019, impondría una tasa del $10 \%$ a productos chinos por valor de 300.000 millones de dólares, con lo que la totalidad de los productos que Estados Unidos importa de China estarían gravados con aranceles (Rappeport, 2019). Posteriormente, en enero de 2020, Estados Unidos y China alcanzaron un principio de acuerdo, que dieron en denominar la "Fase 1" por el que establecieron una tregua en su guerra comercial. Este acuerdo, que entró en vigor el 14 de febrero de 2020, en principio debía ser seguido por una segunda fase en la que se abordarían cuestiones más sensibles como las relacionadas con la propiedad intelectual y el desarrollo tecnológico. Este acuerdo comercial de "Fase 1" parece estar desmoronándose debido a que China no cumple con sus compromisos de comprar productos estadounidenses. Pero, lo que es más importante, el acuerdo de enero guardó silencio sobre algunos de los temas clave que motivaron la guerra comercial de Trump en primer lugar, incluidos los subsidios y protecciones injustas que benefician a las empresas estatales chinas. Todo esto ha quedado en veremos como consecuencia de la pandemia del coronavirus, que ha acentuado la batalla por la preeminencia mundial entre los Estados Unidos y China. Nuevamente, China ha sido blanco de los ataques estadounidenses, que la acusa de haber sido la causante de la pandemia, lo que a su vez ha impedido que se alcance un 
consenso para tratar este tema en el Consejo de Seguridad de las Naciones Unidas (Arredondo, 2020). Sin embargo, al momento de finalizar la redacción de este trabajo, representantes de los Estados Unidos y China mantuvieron una comunicación en la que coincidieron en manifestar que se está progresando en la creación de las infraestructuras gubernamentales necesarias para que el acuerdo sea un éxito y acordaron que, a pesar de la actual pandemia, ambos países esperan cumplir sus obligaciones en virtud del acuerdo de manera oportuna (US Trade Representative, 2020). La etapa que debería seguir es la Fase 2, una discusión de fondo que involucraría el sensible asunto de las patentes y la tecnología. La recesión en la que cayó China en el primer trimestre de 2020 impactó en su protagonismo comercial global y, por tanto, en la actividad de muchos países dependientes de sus compras. Sin embargo, las previsiones económicas indican que los efectos de la pandemia volverían a posicionar a China en la negociación con Estados Unidos. Por el contrario, Trump aparece debilitado para continuar estas negociaciones debido a una crisis sin precedentes, que ha ocasionado que el desempleo suba en los Estados Unidos al 14.7\%, la cifra más alta desde la depresión de 1929 (Thorbecke, 2020).

La OMC prevé una fuerte contracción del comercio mundial para el año en curso que, en un escenario positivo, se sitúa en un $13 \%$ y podría llegar a ser de hasta un $32 \%$, "como consecuencia de la perturbación de la actividad económica normal y de la vida causada por la pandemia de COVID-19 en todo el mundo" (OMC, 2020).

\section{Gráfico 1 - Volumen del comercio mundial de mercancías, 2000-2022}

Índice, $2015=100$

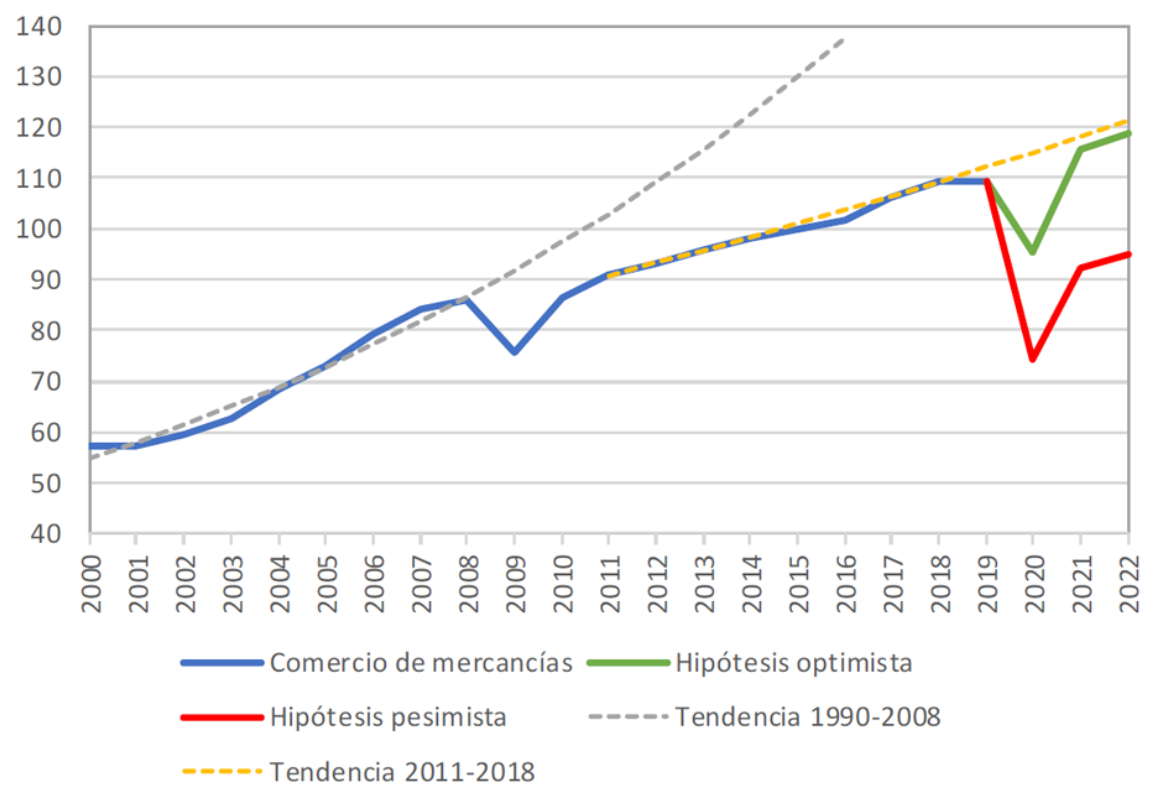

Fuente: $O M C$ 


\section{Revista Peruana de Derecho Internacional \\ Tomo LXX Mayo-Agosto $2020 \mathcal{N}^{\circ}$ 165, pp. 197-225. ISSN: 2663-0222 \\ Recepción: 30/05/2020 Aceptación: 07/07/2020}

La caída del precio del petróleo y de los commodities en general confirman renovadas tensiones que podrían llevar a que se generalicen las guerras comerciales, aunque con nuevos actores, reeditando tensiones del siglo pasado que se creían superadas y que desafían especialmente al sistema multilateral de comercio. En particular, la aplicación de este enfoque unilateralista en la política comercial externa de los Estados Unidos está generando un costo alto de manera creciente a su economía y, en última instancia, podría amenazar la prosperidad mundial. No hay una resolución rápida de esta cuestión a la vista (Ries, 2019).

También la administración se encuentra implicada en otras "guerras comerciales" como, por ejemplo, con la Unión Europea como consecuencia de la aplicación de aranceles comerciales por un valor de 7.500 millones de dólares a determinados productos europeos, que entraron en vigencia el 18 de octubre de 2019 (US Trade Representative, 2019). Si bien estas medidas han sido autorizadas por la OMC, como consecuencia del laudo arbitral por los subsidios ilegales que recibió el fabricante de aviones europeo Airbus ${ }^{6}$, algunos países europeos, como España, ya han anunciado su decisión de aplicar contramedidas contra algunos tradicionales productos de exportación estadounidense, como "las motocicletas Harley Davidson, el whisky 'bourbon' y otros productos similares" (M. C. G. (2019).

\section{El caso de la OMC}

Trump parece haber tenido éxito al ahogar al sistema de solución de diferencias de la Organización Mundial de Comercio (OMC), que se encuentra herido de muerte por el accionar irresponsable de los Estados Unidos, ante la negativa de su administración a renovar los miembros del Órgano de Apelación (OA). El OA se compone de siete miembros y cada diferencia que es apelada es asignada a tres de ellos por rotación (art. 17 del Entendimiento relativo a las normas y procedimientos por los que se rige la solución de diferencias). Estados Unidos ya había bloqueado la sustitución de los cuatro miembros cuyo mandato expiró en 2017, por lo que el OA solo contaba con tres, el número mínimo para funcionar, y el 10 de diciembre de 2019 expiró el mandato de otros dos miembros (del propio Estados Unidos e India) por lo que solo queda solo uno (de China), lo que ha producido una parálisis de facto del sistema de solución de diferencias (Arredondo y Godio, 2019).

Por otra parte, Estados Unidos impone como condición para levantar el bloqueo una reforma de las normas de la OMC alegando una inapropiada utilización del estatus de "país en desarrollo" (White House, 2019), una designación que les permite a los países así categorizados aprovechar ciertos beneficios y exenciones a obligaciones no otorgadas a economías avanzadas, cuando en

\footnotetext{
${ }^{6}$ En una reunión del Órgano de Solución de Diferencias (OSD) celebrada el 14 de octubre de 2019, los Miembros de la OMC acordaron autorizar a los Estados Unidos a imponer contramedidas con respecto al comercio de bienes y servicios de la Unión Europea con los Estados Unidos por valor de hasta USD 7.496.623 millones anuales. La autorización fue concedida de conformidad con la decisión de un árbitro de la OMC emitida el 2 de octubre. Véase caso DS316: Comunidades Europeas y determinados Estados miembros - Medidas que afectan al comercio de grandes aeronaves civiles, disponible en https://www.wto.org/spanish/tratop_s/dispu_s/cases_s/ds316_s.htm (acceso 1/06/2020)
} 


\section{Revista Peruana de Derecho Internacional \\ Tomo LXX Mayo-Agosto $2020 \mathcal{N}^{\circ} 165$, pp. 197-225. ISSN: 2663-0222 \\ Recepción: 30/05/2020 Aceptación: 07/07/2020}

realidad muchos de estos Estados tienen la capacidad de asumir obligaciones plenas. ${ }^{7}$ Dado que la OMC permite la auto-clasificación, no existe una definición universal de quién es un Estado desarrollado o en desarrollo. ${ }^{8}$ El presidente de los Estados Unidos estableció inicialmente un plazo de 90 días para que se avanzara en la reforma de la Organización, tras lo cual, si no se reformaba, procedería unilateralmente a tratar a economías emergentes como países desarrollados (White House, 2019a), algo que todavía no ha ocurrido.

El timing de la decisión de Trump no fue casual, ya que se produjo un día después de que la Unión Europea y Canadá acordaran crear un mecanismo alternativo de solución de diferencias, al que se hace referencia más adelante, ante el riesgo de que el sistema multilateral colapse en caso de que se mantenga el bloqueo de Estados Unidos al nombramiento de integrantes del OA. Esta decisión se ha encontrado con la respuesta de varios países, en particular China y Rusia, que han salido a pronunciarse a favor del multilateralismo y de un orden basado en normas. Asimismo, Alemania y Francia impulsaron una "alianza por el multilateralismo" (Arredondo, 2019), lanzada el 2 de abril de 2019, en el entendimiento de que "la existencia de un orden multilateral basado en el respeto del derecho internacional es la única garantía fiable para la estabilidad internacional y la paz y de que los desafíos a los que nos enfrentamos no pueden resolverse sino a través de la cooperación" (Francia, 2019). ${ }^{9}$

En segundo lugar, la función de la OMC como foro de negociaciones económicas y comerciales multilaterales se ha ido degradando como consecuencia de la dificultad de alcanzar un consenso total entre los miembros, que se requiere para aceptar nuevos acuerdos, obligaciones y cambios de

\footnotetext{
${ }^{7}$ Dos tercios de los 164 Estados miembros de la OMC reclaman el estatus de "país en desarrollo", entre ellos, diez miembros del G-20.
}

${ }^{8}$ La condición de país en desarrollo supone ciertos derechos con relación a las reglas que rigen el comercio internacional, los plazos para la implementación de los acuerdos y, en el ámbito de la asistencia técnica, eleva las oportunidades de acceso a otros mercados. Sin embargo, el hecho de que un Miembro de la OMC decida por sí mismo que es un país en desarrollo no significa que vaya a beneficiarse automáticamente de los programas unilaterales de preferencias de algunos países desarrollados, tales como el Sistema Generalizado de Preferencia (SGP). En la práctica, el país que concede la preferencia es el que decide qué países en desarrollo se beneficiarán de la misma (OMC, 2019). Ello no obstante esta situación es controvertida tanto en doctrina como en la práctica estatal. En el asunto DS246: Comunidades Europeas - Condiciones para la concesión de preferencias arancelarias a los países en desarrollo, el Grupo Especial "constató que: i) la India había demostrado que las preferencias arancelarias concedidas en el marco del Régimen Especial de apoyo a la lucha contra la producción y el tráfico de drogas (el "Régimen Droga") previsto en el esquema SGP de las CE eran incompatibles con el párrafo 1 del artículo I del GATT de 1994; ii) las CE no habían demostrado que el Régimen Droga estuviera justificado de conformidad con el párrafo 2 a) de la Cláusula de Habilitación, que exigía que los beneficios SGP se otorgaran de forma no discriminatoria; y iii) las CE no habían demostrado que el Régimen Droga estuviera justificado de conformidad con el apartado b) del artículo XX del GATT de 1994, puesto que la medida no era "necesaria" para proteger la salud y la vida de las personas en las CE, ni se ajustaba a lo dispuesto en el preámbulo del artículo XX. (Un miembro del Grupo Especial expresó una opinión disidente según la cual la Cláusula de Habilitación no era una excepción al párrafo 1 del artículo I y la India no había formulado ninguna alegación al amparo de la Cláusula de Habilitación.)” (OMC, 2005). El Órgano de Apelación confirmó dos de las constataciones del Grupo Especial: i) la Cláusula de Habilitación funciona como una excepción al párrafo 1 del artículo 1 del GATT de 1994; y ii) la Cláusula de Habilitación no excluye la aplicabilidad del párrafo 1 del artículo 1 del GATT de 1994.

9 Si bien Argentina y Perú no aparecen entre los Estados integrantes de la alianza (cfr. https://multilateralism.org/ministerial-meeting/(acceso 1/06/2020) ), ambos países suscribieron la declaración encabezada por el ministro de Asuntos Exteriores francés y otros 23 ministros para "fortalecer la cooperación global y la solidaridad para combatir el COVID-19" (cfr. https://ar.ambafrance.org/Alianza-para-el-multilateralismodeclaracion-conjunta-de-Jean-Yves-Le-Drian-y). 


\section{Revista Peruana de Derecho Internacional \\ Tomo LXX Mayo-Agosto $2020 \mathcal{N}^{\circ} 165$, pp. 197-225. ISSN: 2663-0222 \\ Recepción: 30/05/2020 Aceptación: 07/07/2020}

reglas. El hecho de que muchos países, incluidas las principales economías, no cumplan con las obligaciones de notificación y transparencia ha dificultado aún más la negociación de nuevas normas y acuerdos. Los Estados Unidos, la Unión Europea y otros han solicitado la adopción de normas que castigarían a los miembros que incumplan con sus obligaciones de transparencia. Sin embargo, es probable que el requisito de consenso presente un obstáculo sustancial para que se acuerden dichas reglas. Ciertos Miembros, incluida China, han aprovechado el punto muerto general en la OMC y continúan manteniendo barreras discriminatorias contra las importaciones, intervienen en los mercados para apoyar a las empresas estatales y no informan los subsidios a la OMC con precisión.

En tercer lugar, algunos países -pero principalmente los Estados Unidos- están preocupados por el sistema de solución de diferencias de la OMC. Específicamente, a los Estados Unidos les preocupa que el OA haya ejercido un poder de decisión y arbitraje que va más allá de su mandato original. ${ }^{10}$ No se trata de una cuestión que haya surgido durante la Administración Trump, sino que sucesivas administraciones estadounidenses han afirmado que el OA, a través de sus resoluciones, aumenta o reduce los derechos de los miembros de la OMC al reinterpretar los acuerdos de la OMC (Misión de los Estados Unidos ante la OMC, 2019). Los restantes Miembros de la OMC nunca han aceptado esas interpretaciones. Si bien el deseo de reforma es compartido por otros Miembros, las opiniones y propuestas de éstos sobre cómo proceder varían considerablemente.

El 11 de febrero de 2020, la Oficina del Representante Comercial de los Estados Unidos (USTR) publicó el Informe sobre el Órgano de Apelación en el que lo acusa de participar en acciones ultra vires y obiter dicta (US Trade Representative 2020a). En dicho informe los Estados Unidos reiteran sus quejan respecto a la actitud de los jueces que, arguye, remueven derechos propios de los Estados Miembros y les agregan nuevas obligaciones, de tal manera que los Estados Unidos no consintieron o procuraron cuando se hicieron miembros de la OMC.

La Administración Trump impone como condición para levantar el bloqueo una reforma de las normas de la OMC alegando que resulta necesario adaptarlas a la realidad de un siglo XXI en el que China, con un sistema basado en subsidios públicos e industrias reguladas, se ha convertido en una gran potencia en los mercados capitalistas, aunque en realidad los motivos son otros (Gao, 2019). Como adecuadamente señala Morales, Estados Unidos

"ha bloqueado la designación de nuevos panelistas como una medida de presión para generar una reforma interna a la $\mathrm{OMC}$, con el propósito de reducir el número de miembros clasificados como 'en desarrollo' y mermar sus beneficios consecuentes, así como aclarar las disposiciones vinculantes en materia judicial, erosionando lo que se perfila como un

\footnotetext{
${ }^{10}$ Las protestas comenzaron en mayo de 2016 (Inside U.S. Trade's, 2019) e incluyeron un caso relacionado con la Argentina: Argentina - medidas relativas al comercio de mercancías y servicios (OMC Doc. WT/DS453/AB/R y Add.1). El día en que se adoptó el informe del OA, 9 de mayo de 2016, Estados Unidos expresó su desacuerdo con las constataciones efectuadas por el OA e hizo una fuerte declaración al respecto. En esa ocasión, el representante de los Estados Unidos expresó que "A los Estados Unidos les preocupa que este enfoque no refleje la función que el ESD atribuye al sistema de solución de diferencias. La función de este sistema no consiste en formular constataciones o interpretaciones jurídicas fuera del contexto de la resolución de una diferencia" y consideró que muchas de "esas declaraciones no son constataciones, sino más bien obiter dicta" (OMC Doc. WT/DSB/M/378).
} 


\section{Revista Peruana de Derecho Internacional \\ Tomo LXX Mayo-Agosto $2020 \mathcal{N}^{\circ}$ 165, pp. 197-225. ISSN: 2663-0222 \\ Recepción: 30/05/2020 Aceptación: 07/07/2020}

sistema de 'justicia' y llevándolo más a uno 'conciliatorio' a través de negociaciones" (Morales, 2020).

La actitud hostil hacia la OMC parece estar ganando cada vez más terreno en los círculos de Washington. A principios de mayo, el senador republicano Josh Hawley presentó un proyecto de resolución conjunta para la retirada de la OMC (Hawley, 2020). Esto fue seguido rápidamente por una propuesta similar en la Cámara de Representantes, presentada por los demócratas Peter DeFazio y Frank Pallone Jr. (DeFazio et al., 2020). Lo preocupante de estos proyectos es que revelan una alineación bipartidista sobre el tema con la administración del presidente Trump, en el Congreso de los Estados Unidos. La denominada "Ley de Acuerdo de la Ronda Uruguay" (P.L.103-465) constituye la base legal para la membresía de los Estados Unidos en la OMC y prevé que la aprobación del Congreso respecto del Acuerdo de la OMC dejará de ser efectiva si el Congreso promulga una resolución conjunta que solicite la retirada. El Congreso puede votar un proyecto de esta naturaleza cada cinco años, siendo ello posible este año 2020. El Senado votó una moción similar en 2005, que DeFazio y Pallone apoyaron. Sin embargo, el Senado nunca adoptó una decisión sobre la retirada de los Estados Unidos de la OMC.

La Unión Europea analizó probables causas de esta "crisis" y propuso opciones de reforma (Unión Europea, 2018). ${ }^{11}$ A medida que transcurría 2019, el sentido de urgencia crecía, pero no se llegó a acordar una solución a este impasse. Durante la reunión del G-20 llevada a cabo en Osaka, los miembros manifestaron su preocupación por la cuestión, a la vez que se mostraron esperanzados en alcanzar una pronta solución a esta cuestión. Por primera vez en una declaración ministerial del G-20 se acordaron detalles de la reforma de la OMC, incluyendo: la importancia de la transparencia de las políticas comerciales, el apoyo a las negociaciones para prohibir ciertos subsidios a la pesca, el reconocimiento a las iniciativas para actualizar las reglas de la OMC, en particular en comercio electrónico, y la necesidad de acción respecto del funcionamiento del sistema de solución de diferencias (G-20, 2019).

A fin de superar este obstáculo al funcionamiento del sistema de solución de diferencias de la OMC, la Unión Europea y Canadá acordaron crear un mecanismo alternativo de solución de diferencias, de naturaleza provisional, basado en las normas vigentes de la OMC (Comisión Europea, 2019). Canadá y la UE recurrirán al arbitraje en virtud del artículo 25 del ESD como un procedimiento de arbitraje de apelación provisional si el OA no puede entender en procedimientos de apelación de informes de grupos especiales debido a un número insuficiente de sus miembros. En tales casos, Canadá y la Unión Europea no presentarán recursos de conformidad con los artículos 16.4 y 17 del ESD. Las partes tienen la intención de replicar lo más fielmente posible todos los aspectos sustantivos y de procedimiento, así como la práctica del OA, de conformidad con el artículo 17 del ESD, incluida la provisión de apoyo administrativo y legal apropiado a los árbitros por parte de la Secretaría del OA. ${ }^{12}$

\footnotetext{
${ }^{11}$ Ver, por ejemplo, el documento conceptual de la Unión Europea (2018). Asimismo, también existe una propuesta de un grupo de 12 miembros de la OMC (2018), recuperado de http://trade.ec.europa.eu/doclib/docs/2018/november/tradoc_157514.pdf. (acceso 1/06/2020)

${ }^{12}$ Véase el texto del acuerdo "Interim Appeal Arbitration Pursuant to Article 25 of the DSU", Bruselas, 25 de julio de 2019, recuperado de http://trade.ec.europa.eu/doclib/docs/2019/july/tradoc_158273.pdf.
} 


\section{Revista Peruana de Derecho Internacional \\ Tomo LXX Mayo-Agosto $2020 \mathcal{N}^{\circ}$ 165, pp. 197-225. ISSN: 2663-0222 \\ Recepción: 30/05/2020 Aceptación: 07/07/2020}

La utilización de este tipo de mecanismos se hizo extensiva a otros Estados Miembros que suscribieron un acuerdo para ello. El 30 de abril de 2020 Australia, Brasil, Canadá, China, Chile, Colombia, Costa Rica, Guatemala, Hong Kong, China; Islandia, México, Noruega, Nueva Zelandia, Pakistán, Singapur, Suiza, Ucrania, la Unión Europea y Uruguay emitieron una "Declaración sobre un mecanismo para elaborar, documentar y comunicar prácticas y procedimientos en la sustanciación de diferencias en la OMC", en la que establecieron un "Procedimiento arbitral multipartito de apelación provisional de conformidad con el artículo 25 del ESD” (Doc. OMC JOB/DSB/1/Add.12).

Para ser justos, algunos de los reproches de los Estados Unidos no son ilegítimos y plantean cuestiones importantes respecto al derecho de la OMC, aunque sus críticas relacionadas con los problemas sistémicos en la solución de diferencias de la OMC, a mi juicio, son desacertadas. Pero, independientemente de la validez de sus reclamos sustantivos, Estados Unidos ha elegido un enfoque inadecuado e incorrecto al mantener como rehén al proceso de nombramiento de integrantes del OA.

Dado que la OMC establece un sistema basado en normas, la existencia de un mecanismo de solución de diferencias reviste fundamental importancia. Si bien, conforme al artículo 3.4 del ESD “(...) las recomendaciones o resoluciones que formule el [Órgano de Solución de Diferencias] OSD tendrán por objeto lograr una solución satisfactoria de la cuestión...", ese mismo artículo en su párrafo segundo subraya que el sistema de solución de diferencias es "(...) un elemento esencial para aportar seguridad y previsibilidad al sistema multilateral de comercio". Por otra parte, este último párrafo prevé que "el sistema sirve para preservar los derechos y obligaciones que los Miembros ... y para aclarar las disposiciones vigentes ... de conformidad con las normas usuales de interpretación del derecho internacional público".

El Informe Anual de la OMC de 2019 subraya que las crecientes tensiones comerciales, el aumento de las medidas de restricción del comercio y la continua incertidumbre económica plantearon verdaderos desafíos al comercio mundial en 2018. Esa situación no ha cambiado, sino que se ha visto agravada por la pandemia del COVID-19 que ha contribuido a que el sistema mundial de comercio continúe enfrentándose a dificultades importantes. Este escenario se ha visto reflejado en el sistema de solución de diferencias de la OMC, al que los miembros han recurrido intensamente para procurar superar sus desavenencias comerciales, habiendo alcanzado el segundo pico más alto en 2018 (OMC, 2019a).

El OA, frecuentemente, ha decidido cuestiones controvertidas, exhibiendo neutralidad frente la agenda neoliberal del comercio reflejada en la Ronda Uruguay y muchos de sus resultados ${ }^{13}$, como los Acuerdos de la OMC sobre los Aspectos de los Derechos de Propiedad Intelectual relacionados con el Comercio (ADPIC) y sobre Obstáculos Técnicos al Comercio (OTC), por ejemplo. Es evidente que los procedimientos fueron exitosos no solo en el sentido de que resolvieron la disputa, sino también porque aclararon importantes cuestiones legales relacionadas con el alcance de las medidas de diversa naturaleza relacionadas con los acuerdos comerciales vigentes. De hecho,

${ }^{13}$ Recuérdese que la Ronda Uruguay fue negociada en el pico de popularidad de las políticas neoliberales representadas por el Consenso de Washington y que comenzaron a ser cuestionadas poco tiempo después sin que la tinta de los Acuerdos de la OMC hubiera tenido la oportunidad de secarse. 
aunque no existe una doctrina del precedente obligatorio (stare decisis) $)^{14}$ en la OMC (Gao, 2019), una característica de su sistema de solución de diferencias es la forma en que los informes del OA, junto con los informes adoptados por los grupos especiales, son especialmente tomados en consideración, generando un acquis que facilita la resolución de casos futuros y contribuye a elucidar las dudas de los miembros respecto de las obligaciones asumidas en el marco de los acuerdos abarcados (Mavroidis, 2008, 421-474).

\section{Conclusiones}

La adopción del Entendimiento relativo a las normas y procedimientos por los que se rige la solución de diferencias en el ámbito de la OMC tuvo una significativa importancia en el plano de las relaciones económicas internacionales y representó un cambio cualitativo en el desarrollo de los sistemas de solución de controversias a nivel mundial.

La interpretación de las normas de la OMC en forma consistente con el derecho internacional y otros principios generales del derecho ha reforzado la seguridad jurídica y le ha brindado coherencia normativa al esquema jurídico de la OMC. Ello hace que el sistema resulte previsible $\mathrm{y}$, por lo tanto, otorga un mayor de seguridad a las partes que quieren recurrir a él para solucionar sus diferencias. La prolijidad de los fallos, la precisión de los razonamientos y la claridad de las opiniones asistirán a las partes cuando se trate de navegar por las complejidades del régimen de la $\mathrm{OMC}$, en particular cuando se trate de aspectos que todavía resultan controvertidos y de cuya interpretación depende el efectivo goce de un derecho.

Asimismo, es posible señalar que la jurisprudencia de la OMC ha efectuado y efectúa una importante contribución a la evolución del derecho internacional contemporáneo, ya que sus decisiones desarrollan la jurisprudencia internacional que es una de las fuentes del derecho internacional. Por ello, la comunidad internacional adquiere mayor seguridad al sentir que un sistema de solución de controversias internacionales puede funcionar basado en principios y de modo coherente y eficaz y, por lo tanto, satisfactorio. La naturaleza judicial del OA le permite ejercer autoridad sobre los Estados Miembros de la Organización, refuerza su condición de sujeto neutral que actúa en forma imparcial y favorece la integridad del sistema. Su posición como juez independiente fortalece su potencialidad para transformarse en una institución internacional legítima y duradera.

Sin embargo, todas las virtudes mencionadas precedentemente no pueden hacernos perder de vista que la OMC experimenta una crisis existencial, que se traduce en un incumplimiento de las funciones para las que fue creada. En primer lugar, el funcionamiento de la organización y, en particular, el de su sistema de solución de diferencias se encuentra bloqueado lo que genera una imposibilidad de administrar eficazmente los acuerdos comerciales abarcados. Por otra parte, la organización "ha fracasado en su capacidad de servir de foro para las negociaciones comerciales multilaterales" (Petrova Georgieva, 2020).

\footnotetext{
${ }^{14}$ La doctrina del precedente obligatorio (stare decisis) consiste en aceptar que el fallo emitido por un tribunal tiene carácter definitivo y sus postulados deben aplicarse a casos similares.
} 
Todavía hay mucho por hacer para mejorar el funcionamiento del OSD de modo que el mismo esté en condiciones de incorporar importantes valores societarios vinculados al comercio en un contexto de una economía cada vez más globalizada e interdependiente. Los Miembros están tratando también de abordar de manera más eficaz las prácticas causantes de distorsión del comercio, como las subvenciones, y de hacer frente a los problemas relativos al OA. El actual estancamiento del proceso de nombramiento de los integrantes del Órgano está ejerciendo una gran presión sobre el sistema de solución de diferencias, por lo que los miembros de la OMC están presentando activamente propuestas para superar ese estancamiento y mejorar el sistema. El OA ya no está en condiciones de entender en los recursos de apelación, por lo que no cabe sino insistir en la importancia de encontrar una solución urgente a esta situación.

En este marco, cuando las cuestiones vinculadas al comercio internacional adquieren una sensibilidad mayor en un escenario altamente conflictivo como el ocasionado por la actual pandemia del COVID-19, donde los valores y disvalores que la globalización trae aparejados son objeto de escrutinio internacional, se torna evidente la necesidad de contar con un ente profesional, justo, imparcial y objetivo que esté en condiciones de resolver las diferencias que puedan surgir entre los distintos componentes del sistema comercial internacional. Los miembros del sistema multilateral de comercio deben hacer frente a estos desafíos de manera inteligente y continuar beneficiándose de su participación en la Organización Mundial de Comercio.

Como señala Shan, "Con el riesgo de una recesión a la vuelta de la esquina, Trump debe reconocer el hecho de que su enfoque actual pone en peligro la economía estadounidense y plantea una amenaza al sistema de comercio internacional, además de que no ha servido para reducir el déficit comercial que tanto aborrece" (Shan, 2020, 124).

Una dura verdad para los Estados Unidos es que ninguna otra economía importante ha mostrado interés en abandonar la OMC. Por lo tanto, si los Estados Unidos decidiera unilateralmente hacerlo quedaría más aislado, con menos capacidad de presionar a China y con un mayor riesgo de quedar relegado económicamente. Aliados de los Estados Unidos como Japón y los países de la Unión Europea han demostrado que están dispuestos a confrontar a China y han propuesto nuevas reglas para abordar la cuestión de los subsidios industriales y la transferencia forzada de tecnología. Pero su enfoque es construir coaliciones de países con ideas afines dentro de la OMC, no abandonar por completo el sistema global basado en reglas e intentar intimidar a China por su cuenta. Lo que los Estados Unidos debería hacer es construir sobre la base que la OMC ya brinda, en lugar de patear el tablero.

\section{Bibliografía}

Alford, Roger P. (2011), “The Self-Judging WTO Security Exception”, Utah Law Review, pp. 697-759, recuperado

de https://scholarship.law.nd.edu/cgi/viewcontent.cgi?article=1336\&context=law_faculty_scholarsh ip. (acceso 1/06/2020)

Arredondo, Ricardo (2020), "La ausencia del Consejo de Seguridad de las Naciones Unidas", Clarín, Buenos Aires, 15 de abril de 2020, recuperado de 
https://www.clarin.com/opinion/ausencia-consejo-seguridad-nacionesunidas_0_0XiPREUyg.html.

Arredondo, Ricardo (2019), "Una alianza para el multilateralismo”, Clarín, Buenos Aires, 20 de septiembre de 2019, recuperado de https://www.clarin.com/opinion/alianzamultilateralismo_0_PEpdlFi7.html.

Arredondo, Ricardo (2018), "La diplomacia del cambio climático", en Sabia de Barberis, Gladys (et. al.), Aspectos ambientales en el derecho internacional, Consejo Argentino para las Relaciones Internacionales (CARI), Buenos Aires, 2018, pp. 61-101.

Arredondo, Ricardo (2017), "Reflexiones en torno al sistema de solución de diferencias de la OMC. Sus implicancias para la Argentina", en Gutiérrez Posse, Hortensia D. T. (et. al.), Temas Jurisdiccionales en materia de Derecho Internacional, Buenos Aires, Consejo Argentino para las Relaciones Internacionales (CARI), 2017.

Arredondo, Ricardo y Godio, Leopoldo M. A. (2019), "La crisis del Órgano de Apelación de la Organización Mundial de Comercio", 13 Revista de la Secretaría del Tribunal Permanente de Revisión (Mercosur), recuperado de http://revistastpr.com/index.php/rstpr/article/view/301/155198.

Bhagwati, Jagdish (2002), "Coping with Antiglobalization: A Trilogy of Discontents”, 81 Foreign Affairs (1), pp. 2-7.

Barshefsky, Charlene; Ross, David J.; van den Broek, Naboth y Hartmann, Stephanie (2019), "WTO Issues Groundbreaking Decision on GATT National Security Exception", WilmerHale, recuperado de https://www.wilmerhale.com/en/insights/client-alerts/20190409-wto-issuesgroundbreaking-decision-on-gatt-national-security-exception.

Baron, Kevin (2020), “Don't Be Fooled. Trump's Cuts to WHO Aren't About the Coronavirus", Defense One, recuperado de https://www.defenseone.com/ideas/2020/04/trumps-cuts-who-arentabout-coronavirus/164631/print/.

Chatzky, Andrew (2019), “Does the New U.S.-Japan Trade Deal Matter?”, Council on Foreign Relations, recuperado de https://www.cfr.org/in-brief/does-new-us-japan-trade-deal-matter.

Comisión Europea (2019), "Joint Statement by the European Union and Canada on an Interim Appeal Arbitration Arrangement", Bruselas, 25 de julio de 2019, recuperado de http://trade.ec.europa.eu/doclib/press/index.cfm?id=2053.

Connaughton, Aidan, Kent, Nicholas y Schumacher, Shannon (2020), "How people around the world see democracy in 8 charts", Pew Research Center, recuperado de https://www.pewresearch.org/fact-tank/2020/02/27/how-people-around-the-world-seedemocracy-in-8-charts/ (acceso 1706/2020).

DeFazio, Peter y Pallone Jr, Frank (2020), "Chairs Peter Defazio and Frank Pallone, Jr. Introduce Legislation to Withdraw The United States from The World Trade Organization", recuperado de https://defazio.house.gov/media-center/press-releases/chairs-peter-defazio-and-frank-pallone-jrintroduce-legislation-to 
Evenett, Simon J. y Fritz, Johannes (2019), Jaw not War: Prioritising WTO Reform Options. The 24th Global Trade Alert Report, CEPR Press, 2019, p. 6, recuperado de https://www.globaltradealert.org/reports/download/47.

Francia (2019), "Alianza por el Multilateralismo", recuperado de https://www.diplomatie.gouv.fr/es/politica-exterior/francia-en-naciones-unidas/alianza-por-elmultilateralismo/article/alianza-por-el-multilateralismo.

Foot, Rosemary, MacFarlane, Neil y Mastanduno, Michael (2003), U.S. Hegemony and International Organizations. The United States and Multilateral Institutions, Oxford, Oxford University Press.

G-20 (2020), Osaka Leaders' Declaration, Osaka, Japón, 29 de junio de 2019, en particular el parágrafo $8, \quad$ recuperado de https://www.wto.org/english/news_e/news19_e/g20_osaka_leaders_declaration_e.pdf.

Galbraith, Jean (2018), "Contemporary Practice of the United States Relating to International Law: United States Withdraws from the UN Human Rights Council, Shortly After Receiving Criticism About Its Border Policy", 112 The American Journal of International Law (4), pp. 745-751.

Gao, Henry S. (2019), "Disruptive Construction or Constructive Destruction? Reflections on the Appellate Body Crisis", en Chang-fa Lo, Jinji Nakagawa y Tsai-yu Lin (eds.), The Appellate Body of the WTO and Its Reform, Springer, recuperado de https://papers.ssrn.com/sol3/papers.cfm?abstract_id=3422025.

Goldsmith, Jack y Mercer, Shannon Togawa (2018), "International Law and Institutions in the Trump Era", 61 German Yearbook of International Law, recuperado de https://papers.ssrn.com/sol3/Delivery.cfm/SSRN_ID3324582_code647786.pdf?abstractid=33245 $82 \&$ mirid $=1$.

Hawley, Josh (2020), "Withdrawing approval of the Agreement Establishing the World Trade", recuperado de https://www.hawley.senate.gov/sites/default/files/2020-05/Hawley-WTOResolution.pdf.Organization",

Inside U.S. Trade's, recuperado de https://insidetrade.com/daily-news/debate-erupts-over-usblocking-korean-appellate-body-reappointment.

Khaler, Miles (2017), "President Trump and the Future of Global Governance", Council on Foreign Relations, recuperado de https://www.cfr.org/blog/president-trump-and-future-globalgovernance.

Koh, Harold Hongju (2019), The Trump Administration and International Law, New York: Oxford University Press.

Landler, Mark y Rappeport, Alan (2018), "Trump Hails Revised Nafta Trade Deal, and Sets Up a Showdown With China", The New York Times, recuperado de https://www.nytimes.com/2018/10/01/us/politics/nafta-deal-trump-canada-mexico.html.

Lester, Simon, Manak, Inu y Kim, Kyounghwa (2019), “Trump's First Trade Deal: The Slightly Revised Korea-U.S. Free Trade Agreement”, CATO Free Trade Bulletin N $^{\circ} 73$, recuperado de 
https://www.cato.org/publications/free-trade-bulletin/trumps-first-trade-deal-slightly-revisedkorea-us-free-trade.

M. C. G. (2019), "Entran en vigor los aranceles de Trump a la Unión Europea por valor de 7.500 millones", Expansión, 18 de octubre de 2019, disponible en https://www.expansion.com/economia/2019/10/18/5da94db4468aeb2c028b4690.html.

Misión de los Estados Unidos ante la OMC (2019), "Statements by the United States at the Meeting of the WTO Dispute Settlement Body", 25 de febrero de 2019, p. 12, recuperado de https://geneva.usmission.gov/wp-content/uploads/sites/290/Feb25.DSB_.Stmt_.asdeliv.fin_.public.pdf.

Morales, Manuel (2020), "Estados Unidos critica poder del Órgano de Apelación de la OMC”, El Economista, El Economista, recuperado de https://www.eleconomista.com.mx/empresas/EstadosUnidos-critica-poder-del-Organo-de-Apelacion-de-la-OMC-20200414-0036.html.

Mavroidis, Petros C. (2008), "No Outsourcing of Law? WTO Law as Practiced by WTO Courts", 102 American Journal of International Law (3).

Noack, Rick (2020), "After Trump suspends payments to WHO, other countries rally behind the agency", The Washington Post, recuperado de https:/www.washingtonpost.com/world/aftertrump-suspends-payments-to-who-other-countries-rally-behind-theagency/2020/04/15/1a2ec7c6-7f0e-11ea-84c2-0792d8591911_story.html.

OEA-SICE (2019), “Acuerdo Amplio y Progresista de Asociación Transpacífico (CPTPP)) Australia, Brunéi Darussalam, Canadá, Chile, Japón, Malasia, México, Nueva Zelandia, Perú, Singapur y Vietnam”, recuperado de http://www.sice.oas.org/TPD/TPP/TPP_s.ASP.

OMC (2020), "Desplome del comercio ante la pandemia de COVID-19, que está perturbando la economía mundial", recuperado de https:/www.wto.org/spanish/news_s/pres20_s/pr855_s.htm.

OMC (2019), “¿Qué países se consideran países en desarrollo en la OMC?”, recuperado de https://www.wto.org/spanish/tratop_s/devel_s/d1who_s.htm.

OMC (2019a), Informe Anual 2019, Ginebra, 2019, p. 124 y ss., recuperado de https://www.wto.org/spanish/res_s/publications_s/anrep19_s.htm.

OMC (2005), "DS246: Comunidades Europeas - Condiciones para la concesión de preferencias arancelarias a los países en desarrollo", recuperado de https://www.wto.org/spanish/tratop_s/dispu_s/cases_s/ds246_s.htm.

Pearson, Federic S. y Rochester, J. Martin (2000), Relaciones Internacionales. Situación global en el siglo XXI, $4^{\mathrm{a}}$ ed., Bogotá: McGraw-Hill.

Petrova Georgieva, Virdzhiniya (2020), "La crisis de la Organización Mundial del Comercio: problemas e (im) posibles soluciones”, Anuario Mexicano de Derecho Internacional, vol. XX, pp. 25-60.

Rappeport, Alan (2019), "Trump Says U.S. Will Hit China With More Tariffs", The New York Times, recuperado de https://www.nytimes.com/2019/08/01/us/politics/china-trade-trump.html. 
Reinsch, William Alan y Caporal, Jack (2019), “The WTO's First Ruling on National Security: What Does It Mean for the United States?", Center for Strategic and International Studies, recuperado de https://www.csis.org/analysis/wtos-first-ruling-national-security-what-does-itmean-united-states.

Ries, Charles P. (2019), “Trump's Tariffs Against China Aren't Working. And There's No Quick Resolution in Sight”, Los Angeles Times, 20 de agosto de 2019.

Sanahuja, José Antonio (2020), ¿Bipolaridad en ascenso?”, 20 Foreign Affairs Latinoamerica (2).

Scarborough, Joe (1995), H.R. 2535 (IH) - United Nations Withdrawal Act of 1995, recuperado de https://www.govinfo.gov/app/details/BILLS-104hr2535ih.

Shan, Weijian (2020), "La guerra comercial imposible de ganar. En el enfrentamiento entre China y Estados Unidos, pierden sobre todo los estadounidenses", 20 Foreign Affairs Latinoamerica (2).

Slobodian, Quinn (2018), Globalists. The End of Empire and the Birth of Neoliberalism, Cambridge, Massachusetts, Harvard University Press.

Tamir, Yael (2020), "La construcción de un nacionalismo mejor: El lugar de las naciones en un mundo globalizado", 20 Foreign Affairs Latinoamerica (2), pp. 93-97.

The American Presidency Project (1984), Republican Party Platform of 1984, recuperado de https://www.presidency.ucsb.edu/documents/republican-party-platform-1984.

Thorbecke, Catherine (2020), "US unemployment rate skyrockets to $14.7 \%$, the worst since the Great Depression", $A B C$ News, recuperado de https://abcnews.go.com/Business/us-economy-lost205-million-jobs-april-unemployment/story?id=70558779.

Trump, Donald J. (2016), "Full transcript: Donald Trump's jobs plan speech", Politico, 28 de julio de 2016, recuperado de https://www.politico.com/story/2016/06/full-transcript-trump-job-planspeech-224891.

Trump, Donald J. (2018), Twitter, 12 April 2018, 8:15 PM, recuperado de https://twitter.com/realDonaldTrump/status/984631073865953280.

Unión Europea (2018), Concept paper: WTO modernisation, recuperado de http://trade.ec.europa.eu/doclib/docs/2018/september/tradoc_157331.pdf.

US Department of State (2018), "Remarks on the UN Human Rights Council", recuperado de https://www.state.gov/remarks-on-the-un-human-rights-council/ (acceso 7/11/2019).

US Department of State (2019), "Department Press Briefing - March 26, 2019”, recuperado de https://www.state.gov/briefings/department-press-briefing-march-26-2018.

US Trade Representative (2019), “U.S. Wins \$7.5 Billion Award in Airbus Subsidies Case”, 2 de octubre de 2019, recuperado de https://ustr.gov/about-us/policy-offices/press-office/pressreleases/2019/october/us-wins-75-billion-award-airbus. 
US Trade Representative (2020), "USTR and Treasury Statement on Call With China", recuperado de $\quad$ https://ustr.gov/about-us/policy-offices/press-office/press-releases/2020/may/ustr-andtreasury-statement-call-china.

US Trade Representative (2020a), "Report on The Appellate Body of The World Trade Organization", recuperado de https://ustr.gov/sites/default/files/Report_on_the_Appellate_Body_of_the_World_Trade_Organi zation.pdf

Vásquez Arango, Carolina (2011), "La jurisprudencia sobre las excepciones relativas a la seguridad en el acuerdo GATT", 2 Forum, recuperado de https://dialnet.unirioja.es/descarga/articulo/3924377.pdf.

White House (2017), "Presidential Memorandum Regarding Withdrawal of the United States from the Trans-Pacific Partnership Negotiations and Agreement", recuperado de https://www.whitehouse.gov/presidential-actions/presidential-memorandum-regardingwithdrawal-united-states-trans-pacific-partnership-negotiations-agreement/.

White House (2017), "The Inaugural Address", Washington DC, recuperado de https://www.whitehouse.gov/briefings-statements/the-inaugural-address/.

White House (2018), "Remarks by President Trump to the 73rd Session of the United Nations General Assembly", New York, NY, recuperado de https://www.whitehouse.gov/briefingsstatements/remarks-president-trump-73rd-session-united-nations-general-assembly-new-yorkny/.

White House (2019), Memorandum on Reforming Developing-Country Status in the World Trade Organization, recuperado de https://www.whitehouse.gov/presidential-actions/memorandumreforming-developing-country-status-world-trade-organization/.

White House (2019a), Memorandum on Reforming Developing-Country Status in the World Trade Organization, 26 de julio de 2019, recuperado de https://www.whitehouse.gov/presidentialactions/memorandum-reforming-developing-country-status-world-trade-organization/. 